\title{
EDITORIAL
}

\section{Antimicrobial peptides in the real world: implications for cystic fibrosis}

\author{
P.S. Hiemstra
}

$\mathbf{T}$ he continuous cycle of respiratory infections and lung inflammation is a hallmark of cystic fibrosis (CF) lung disease and plays a central role in respiratory failure that develops in end-stage disease. Effective mucociliary clearance is an essential mechanism in host defence against infection and requires a functional "two-layer" mucus clearance system composed of a periciliary liquid layer and an overlying mucus layer in which inhaled substances are trapped [1]. Together these two separate layers constitute airway surface liquid (ASL). Adequate hydration is essential for an optimal function of the mucociliary clearance system. Dehydration of the mucosal surface in CF may not only hamper effective mucus clearance but also causes adhesion of mucus to the epithelial surface. This results in airway flow obstruction, inflammation and favouring infections.

In addition to this defect in mucociliary clearance, an inadequate function of the secreted effector molecules of the innate immune system in the lung has been suggested to contribute to increased susceptibility to infection in CF [2]. Antimicrobial peptides and proteins are an essential element of this innate immune system, and the airway epithelium forms a range of these compounds [3]. The main classes of antimicrobial peptides produced by airway epithelial cells are the $\beta$-defensins and the cathelicidins (LL-37 in humans). The antimicrobial activity of most of these peptides is markedly restricted in isotonic and hypertonic conditions, and optimal activity under laboratory conditions is observed in hypotonic conditions. In 1996, SMITH et al. [4] demonstrated that the ASL secreted by cultured airway epithelial cells from CF patients is deficient in antimicrobial activity as a result of increased salt concentrations. Subsequent studies showed that the activity of antimicrobial peptides, such as $\beta$-defensins, is salt-dependent and, therefore, may be reduced in ASL collected from CF airway epithelial cells [5]. However, in contrast to cell cultures, the actual salt concentration of ASL in the lung is very difficult to assess. Therefore, it is still unknown whether normal ASL is hypotonic and whether ASL in CF is characterised by increased salt concentrations. Using a bronchial xenograft system, BALS et al. [6] demonstrated that removal of salt from

*Dept of Pulmonology, Leiden University Medical Center, Leiden, The Netherlands.

STATEMENT OF INTEREST: None declared.

CORRESPONDENCE: P.S. Hiemstra, Dept of Pulmonology, Building 1 C3-P, Leiden University Medical Center, P.0. Box 9600, 2300 RC Leiden, The Netherlands. Fax: 31 715266927. E-mail: p.s.hiemstra@lumc.nl
ASL collected from both normal and CF epithelial cells increases antimicrobial activity but also noted that the activity of this desalted CF ASL is markedly lower when compared with desalted normal ASL. Nevertheless, concentrations of known antimicrobial peptides and proteins did not differ between CF and normal ASL. These data suggest that the activity of antimicrobial peptides in CF ASL is blocked in a salt-independent fashion. One possibility is that antimicrobial peptides are proteolytically degraded in $\mathrm{CF}$, as shown by the observation that human $\beta$-defensins 2 and 3 are inactivated by cathepsins B, L and S [7]. These cysteine proteases are present in increased concentrations in the lungs of patients with inflammatory lung diseases, such as CF.

In the current issue of the European Respiratory Journal, BUCKI et al. [8] present another explanation for decreased antimicrobial activity at the epithelial surface in CF. They demonstrated the presence of LL-37 in CF sputum bound to components such as DNA, F-actin and cellular debris. LL-37 is the only cathelicidin antimicrobial peptide produced in humans and is synthesised as the precursor human (h)CAP18. hCAP18 is processed by proteolytic cleavage resulting in the release of the antimicrobial peptide LL-37. This cationic $\alpha$-helical peptide composed of 37 amino acids, from which it derives its name, has marked antimicrobial and lipopolysaccharide (LPS) neutralising activities. In addition, it is involved in inflammation (partly through its activity as a chemoattractant, for example for neutrophils), immunity and wound repair [9]. It is mainly produced by neutrophils but also by airway epithelial cells and other cell types.

In vivo epithelial antimicrobial peptides are released onto the apical surface of airway epithelial cells into a complex environment, which is continuously subject to change in composition and properties: the real world. This environment is markedly different from the well-defined buffers and media that are mostly used for analysis of antimicrobial properties of these peptides. The ASL into which the peptides are released is composed of two layers: 1 ) the periciliary layer around the cilia and the microvilli which contains cell-surface tethered mucins; and 2) the overlying mucus layer, which contains the secreted, gel-forming mucins [1]. The distribution of released antimicrobial peptides between the two layers that constitute the ASL is unknown. Several components that are present in ASL of normal and inflamed lungs may restrict the antimicrobial activity of antimicrobial peptides, including: mucins [10], bacterial polysaccharides released by lung pathogens [11] and DNA and F-actin [12]. This DNA and F-actin is present in increased 
amounts in airway secretions in CF, and it is most likely that it is released from neutrophils and other inflammatory cells. BUCKI et al. [8] show that components that dissolve the DNA/F-actin bundles, such as DNAse I and gelsolin, release LL-37 from the complexes composed of F-actin, DNA and LL-37, which are present in CF sputum. Furthermore, BUCKI et al. [8] show that LL-37 in CF sputum may be inactivated by the presence of LPS released from bacteria, such as Pseudomonas aeruginosa, and demonstrate that an LPS-binding peptide may prevent this inactivation. LL-37 itself also binds LPS and is known to inactivate the pro-inflammatory activity of LPS and, therefore, will compete with the LPS-binding peptide for binding to LPS. BUCKI et al. [8] conclude that a combination of components which dissolve DNA/F-actin aggregates and substances that bind LPS may be considered as a novel therapeutic approach for the treatment of chronic respiratory infections in $\mathrm{CF}$.

The study by BUCKI et al. [8] highlights the role of the antimicrobial peptide LL-37 in CF lung disease and demonstrates that the activity of this endogenous antibiotic can be enhanced by release from DNA/F-actin bundles and inhibiting LPS. It is likely that the observations described in that paper for LL-37 may also be relevant for other cationic antimicrobial peptides and proteins. Whereas the study of BUCKI et al. [8] provides further insight into the defective innate immunity in $\mathrm{CF}$, another intriguing explanation for such a defect was provided in a recent study by MoskwA et al. [13]. These authors showed that the CF transmembrane conductance regulator (CFTR) chloride channel in airway epithelial cells is involved in the formation of antimicrobial hypothiocyanate, which is generated through formation of reactive oxygen intermediates. CF is caused by mutations in CFTR and, therefore, the finding that this pathway is defective in CF airway epithelial cells sheds new light on the breach in innate immunity that may underlie susceptibility to respiratory infections in CF. Therefore, multiple pathways may be defective in the innate immune system in the CF lung.

In view of the high degree of synergy between the various antimicrobial mechanisms in the lung, it is likely that various therapeutic strategies can be employed to increase the host defence in cystic fibrosis. This is especially important because of the rapid emergence of Pseudomonas strains that are resistant to treatment with conventional antibiotics.

\section{REFERENCES}

1 Randell SH, Boucher RC, University of North Carolina Virtual Lung Group. Effective mucus clearance is essential for respiratory health. Am J Respir Cell Mol Biol 2006; 35: 20-28.

2 Bals R, Weiner DJ, Wilson JM. The innate immune system in cystic fibrosis lung disease. J Clin Invest 1999; 103: 303-307.

3 Bals R, Hiemstra PS. Innate immunity in the lung: how epithelial cells fight against respiratory pathogens. Eur Respir J 2004; 23: 327-333.

4 Smith JJ, Travis SM, Greenberg EP, Welsh MJ. Cystic fibrosis airway epithelia fail to kill bacteria because of abnormal airway surface fluid. Cell 1996; 85: 229-236.

5 Goldman MJ, Anderson GM, Stolzenberg ED, Kari UP, Zasloff M, Wilson JM. Human $\beta$-defensin- 1 is a saltsensitive antibiotic in the lung that is inactivated in cystic fibrosis. Cell 1997; 88: 553-560.

6 Bals R, Weiner DJ, Meegalla RL, Accurso F, Wilson JM. Salt-independent abnormality of antimicrobial activity in cystic fibrosis airway surface fluid. Am J Respir Cell Mol Biol 2001; 25: 21-25.

7 Taggart CC, Greene CM, Smith SG, et al. Inactivation of human beta-defensins 2 and 3 by elastolytic cathepsins. J Immunol 2003; 171: 931-937.

8 Bucki R, Byfield FJ, Janmey PA. Release of the antimicrobial peptide LL-37 from DNA/F-actin bundles in cystic fibrosis sputum. Eur Respir J 2007; 29: 624-632.

9 Tjabringa GS, Rabe KF, Hiemstra PS. The human cathelicidin LL-37: a multifunctional peptide involved in infection and inflammation in the lung. Pulm Pharmacol Ther 2005; 18: 321-327.

10 Felgentreff $K$, Beisswenger $C$, Griese $M$, Gulder $T$, Bringmann G, Bals R. The antimicrobial peptide cathelicidin interacts with airway mucus. Peptides 2006; 27: 3100-3106.

11 Herasimenka $Y$, Benincasa $M$, Mattiuzzo M, Cescutti $P$, Gennaro R, Rizzo R. Interaction of antimicrobial peptides with bacterial polysaccharides from lung pathogens. Peptides 2005; 26: 1127-1132.

12 Weiner DJ, Bucki R, Janmey PA. The antimicrobial activity of the cathelicidin LL37 is inhibited by F-actin bundles and restored by gelsolin. Am J Respir Cell Mol Biol 2003; 28:738-745.

13 Moskwa P, Lorentzen D, Excoffon KJ, et al. A novel host defense system of airways is defective in cystic fibrosis. Am J Respir Crit Care Med 2007; 175: 174-183. 